Laser Chem., 1999, Vol. 19, pp. 75-78

Reprints available directly from the publisher Photocopying permitted by license only
(C) 1999 OPA (Overseas Publishers Association) N.V. Published by license under the Harwood Academic Publishers imprint, part of The Gordon and Breach Publishing Group.

Printed in India.

\title{
PUMP- AND PROBE-WAVELENGTH DEPENDENCIES OF PICOSECOND ANTI-STOKES RAMAN SPECTRUM OF TRANS-STILBENE IN THE $\mathrm{S}_{1}$ STATE
}

\author{
TAKAKAZU NAKABAYASHI*, HIROMI OKAMOTO \\ and MITSUO TASUMI ${ }^{\dagger}$
}

Department of Chemistry and Research Centre of Spectrochemistry, School of Science, The University of Tokyo, Bunkyo-ku, Tokyo 113, Japan

(Received 6 April 1997)

Vibrational relaxation dynamics of trans-stilbene in the $\mathrm{S}_{1}$ state immediately after photoexcitation is studied by picosecond time-resolved anti-Stokes Raman spectroscopy with several pump and probe wavelengths. Pump-wavelength dependence of the antiStokes spectrum indicates that, when pump photons with high excess energy $\left(\approx 5200 \mathrm{~cm}^{-1}\right)$ are used, the anti-Stokes Raman bands at $0 \mathrm{ps}$ delay time arise from vibrationally excited transients with excess vibrational energy not thermally distributed in the molecule. Probe-wavelength dependence suggests that the vibrationally excited transients at 0 ps are mostly on the lowest excited vibrational levels, as far as the olefinic $\mathrm{C}=\mathrm{C}$ stretching and the $\mathrm{C}-\mathrm{Ph}$ stretching modes are concerned. The vibrational relaxation process of $S_{1}$ trans-stilbene is discussed on the basis of the observed results.

Keywords: anti-Stokes Raman scattering; time-resolved Raman spectroscopy; resonance Raman excitation profiles; vibrational relaxation; trans-stilbene

Time-resolved anti-Stokes Raman spectroscopy is very useful for studies of vibrational relaxation dynamics in solution, since it probes only those molecules which are populated in excited vibrational states, giving mode-specific information [1, 2]. In the present work, we show that pump- and probe-wavelength dependencies of the anti-Stokes

\footnotetext{
* Corresponding author.

${ }^{\dagger}$ Present address: Department of Chemistry, Faculty of Science, Saitama University, Urawa, Saitama 338, Japan.
} 
Raman spectra provide important information on the vibrational relaxation dynamics of trans-stilbene in the excited singlet $\left(\mathrm{S}_{1}\right)$ state.

Figure 1 shows the pump-wavelength dependence of the anti-Stokes Raman spectra of $\mathrm{S}_{1}$ trans-stilbene at $0 \mathrm{ps}$ delay time. The intensity of the $\nu_{1}$ (olefinic $\mathrm{C}=\mathrm{C}$ stretch) band relative to that of the $\nu_{6}(\mathrm{C}-\mathrm{Ph}$ stretch) band shows a clear pump-wavelength dependence: the $\nu_{1}$ band in the 277 -nm pump spectrum (excess vibrational energy $\approx 5200 \mathrm{~cm}^{-1}$ ) is much stronger than that in the $320 \mathrm{~nm}$ pump spectrum (excess energy $\approx 400 \mathrm{~cm}^{-1}$ ). Anti-Stokes band intensities in the $320 \mathrm{~nm}$ pump spectrum are independent of the delay time up to $20 \mathrm{ps}$. On the other hand, the intensities of all the bands in the $277 \mathrm{~nm}$ pump spectrum decrease with a time constant of $\approx 10$ ps. Relative intensities among bands also change in a similar time scale. Such temporal behaviour can be attributed to vibrational relaxation toward a thermal equilibrium. Spectral features of the $277 \mathrm{~nm}$ pump spectrum at $20 \mathrm{ps}$ are similar to

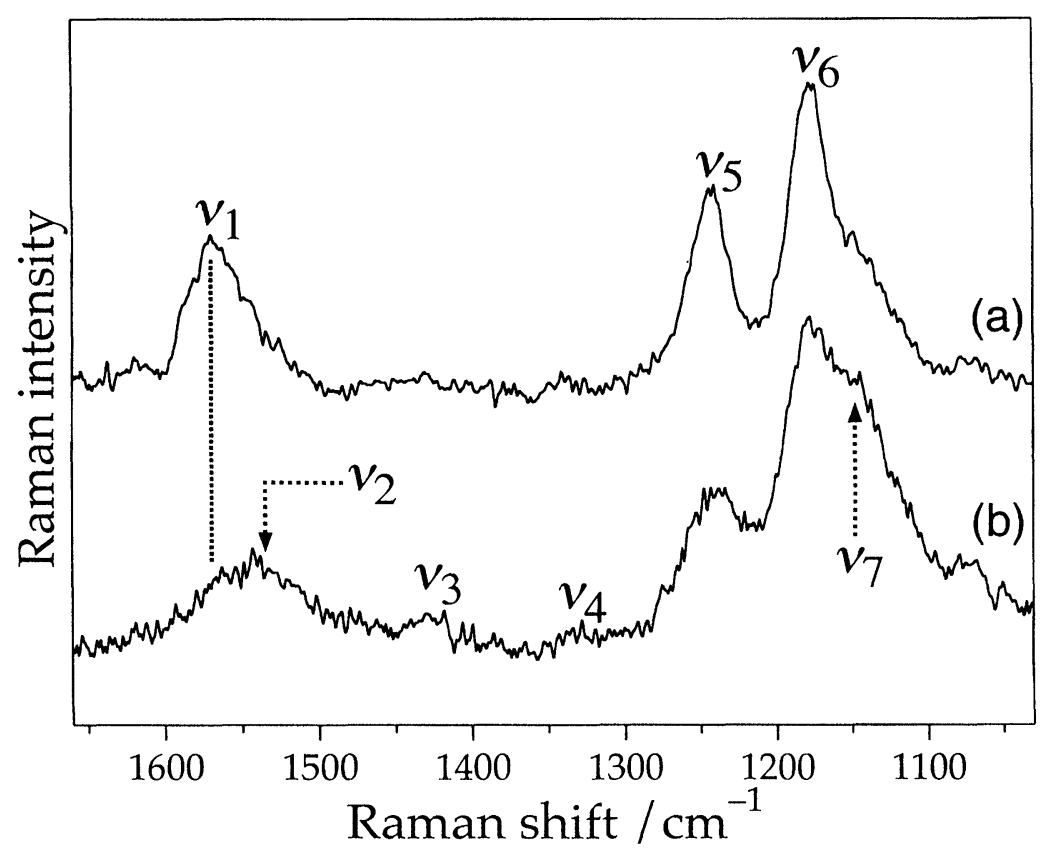

FIGURE 1 Anti-Stokes Raman spectra of trans-stilbene in $\mathrm{S}_{1}$ state at $0 \mathrm{ps}$ delay time in a 1-butanol solution. Pump wavelength, (a) 277 and (b) $320 \mathrm{~nm}$; probe wavelength, $610 \mathrm{~nm}$. Each spectrum is normalised to the intensity of the $\nu_{6}$ band at $1180 \mathrm{~cm}^{-1}$. 
those of the $320 \mathrm{~nm}$ pump spectrum. The intensity of the $\nu_{6}$ band relative to that of the $\nu_{7}$ band, and the intensity of the $\nu_{1}$ band relative to that of the $\nu_{2}$ band at 0 ps show marked changes with the pump wavelength. This observation cannot be explained if the excess energy is statistically distributed among all the vibrational modes, and indicates that the excess energy taken from the $277 \mathrm{~nm}$ photon is localised in the $\nu_{1}$ and $\nu_{6}$ modes at 0 ps delay time.

Next, the probe-wavelength dependence of the band intensities at 0 ps was recorded using the $277 \mathrm{~nm}$ pump, and compared with simulated anti-Stokes resonance Raman excitation profiles (REPs), in order to examine the vibrational quantum numbers of the $\nu_{1}$ and $\nu_{6}$ modes. The simulation was based on the A-term of Albrecht's formula. Parameters used for the simulation were obtained from an analysis of the Stokes REPs and the $\mathrm{S}_{n} \leftarrow \mathrm{S}_{1}$ absorption spectrum. The anti-Stokes Raman spectra of $S_{1}$ trans-stilbene were observed with eight probe wavelengths $(580-670 \mathrm{~nm})$, all of which were resonant or pre-resonant with the strong $\mathrm{S}_{n} \leftarrow \mathrm{S}_{1}$ absorption. We introduce a quantity $r(\lambda)\left[\equiv\left(I_{6}(\lambda) / I_{1}(\lambda)\right) \times\left(I_{1}\left(\lambda_{0}\right) / I_{6}\left(\lambda_{0}\right)\right)\right.$, where $I_{i}(\lambda)$ denotes the observed anti-Stokes Raman intensity of the $\nu_{i}$ band for probe wavelength $\lambda$, and $\lambda_{0}$ is a fixed wavelength used for reference], which represents a normalised intensity ratio, and we compare the calculated and observed $r(\lambda)$ 's. The simulated $r(\lambda)$ 's are characteristic of individual initial levels. The observed points are best reproduced if we assume that both the $\nu_{1}$ and $\nu_{6}$ bands arise from the lowest vibrationally excited levels of the respective modes. This result indicates that the vibrationally excited transients at $0 \mathrm{ps}$ are mostly on the lowest excited vibrational levels, as far as the $\nu_{1}$ and $\nu_{6}$ modes are concerned. It is further shown from the analysis of the anti-Stokes REPs that the $277 \mathrm{~nm}$ pump spectrum at 0 ps can be quantitatively explained only when a nonthermal distribution of the excess energy is assumed. This is in accordance with the pump-wavelength dependence results. Considering the temporal resolution $(5-7 \mathrm{ps})$, it is concluded that it takes at least several picoseconds to reach an intramolecular thermal equilibrium in $\mathrm{S}_{1}$ trans-stilbene.

From the present study based on the analysis of anti-Stokes REPs, the intramolecular vibrational relaxation process is considered to proceed in roughly two steps. The optically pumped molecules on highly excited vibrational levels of the Franck - Condon active (high- 
frequency) modes first relax very rapidly (in the femtosecond regime) to the lowest vibrationally excited state, and yet the excess vibrational energy is not statistically distributed. In the next step of the relaxation (in several picoseconds), an intramolecular (quasi-)thermal equilibrium is reached. The vibrational cooling follows after that. The present result suggests that, even in a large molecule such as transstilbene, there is a bottleneck state in the intramolecular vibrational relaxation in solution [3].

\section{References}

[1] Schultz, S. L., Qian, J. and Jean, J. M. (1997). J. Phys. Chem. A, 101, 100.

[2] Nakabayashi, T., Okamoto, H. and Tasumi, M., J. Phys. Chem. in press.

[3] Owrutsky, J. C., Raftery, D. and Hochstrasser, R. M. (1994). Annu. Rev. Chem., 45, 519. 